\title{
Long-term insulin treatment of 3T3-L1 adipocytes results in mis-targeting of GLUT4: implications for insulin-stimulated glucose transport
}

\author{
V. H.Maier, G. W. Gould \\ Division of Biochemistry and Molecular Biology, Davidson Building, Institute of Biomedical + Life Sciences, \\ University of Glasgow, Glasgow, G12 8QQ, Scotland
}

\begin{abstract}
Aims/hypothesis. Insulin stimulates glucose transport in adipose and muscle tissue by the translocation of a specialised pool of intracellular GLUT4-containing vesicles to the cell surface. It is well established that defective insulin-stimulated GLUT4 translocation is associated with insulin resistance. Long-term insulin treatment $(500 \mathrm{nmol} / \mathrm{l}$ for $24 \mathrm{~h})$ of 3T3-L1 adipocytes has previously been shown to decrease cellular GLUT4 content and reduce insulin-stimulated GLUT4 translocation. Here, we test the hypothesis that the insulin resistance observed after long-term insulin treatment arises by the selective loss of GLUT4 from a specific intracellular compartment.

Methods. Using iodixanol gradient centrifugation we have separated intracellular GLUT4 containing membranes into two distinct populations corresponding to recycling endosomes and a distinct intracellular compartment which probably represents GLUT4 storage vesicles (GSVs).
\end{abstract}

Results. A short-term insulin stimulation reduced the content of GLUT4 in the GSV fraction $(51 \pm 3.5 \%)$ with only a modest decrease from the endosomal fraction $(23 \pm 2.6 \%)$. Long-term insulin treatment decreased cellular GLUT4 content by about $40 \%$ and diminished the ability of a short-term insulin challenge to promote GLUT4 translocation. We further show that this depletion of cellular GLUT4 is selectively from the GSV fraction $(68 \pm 7 \%$ decrease compared to untreated cells).

Conclusions/interpretation. Such data argue that long-term insulin treatment results in the mis-targeting of GLUT4 such that it no longer accesses the GSV compartment. These data imply that defective targeting of GLUT4 away from the GSV compartment plays an important role in the aetiology of insulin resistance. [Diabetologia (2000) 43: 1273-1281]

Keywords Glucose transport, GLUT4, endosome, insulin resistance, insulin.
Insulin stimulates glucose transport in muscle and adipose cells by virtue of the expression of a unique glucose transporter, GLUT4. Unlike other GLUT iso-

Received: 22 March 2000 and in revised form: 31 May 2000

Corresponding author: G. W. Gould, Division of Biochemistry and Molecular Biology, Davidson Building, University of Glasgow, Glasgow, G12 8QQ, Scotland

Abbreviations: GLUT, glucose transporter; IRAP, insulin-responsive aminopeptidase; TGN, trans-Golgi network; TfR, transferrin receptor; GSV, GLUT-4 storage vesicle; PM, plasma membrane; LDM, low-density microsomes; HDM, highdensity microsomes; VAMP2, vesicle-associated membrane protein 2 . forms, in the absence of insulin more than $95 \%$ of GLUT4 is intracellularly sequestered but translocates rapidly to the cell surface in response to insulin stimulation [1]. Although other molecules such as the transferrin receptor (TfR) and GLUT1 also undergo insulin-dependent movement to the cell surface, the magnitude of these effects is much smaller than that of GLUT4 ( twofold increase compared with $\sim 15$ to 20 -fold, respectively) [2,3], a difference which seems to be due to a unique intracellular trafficking event involving the sorting of GLUT4 from other endosomal traffic. Although a proportion of GLUT4 is found in endosomes and the TGN [4,5] a significant proportion $(\sim 60 \%)$ is localised to a dis- 
crete vesicle pool termed GLUT4 storage vesicles (GSVs) $[6,7,8,9]$. This discrete vesicle pool could represent GLUT4 within a specialised intracellular compartment or GLUT4 localised within a slowly recycling subdomain of the endosomal system. The major function of this distinct intracellular GLUT4 compartment seems to be to sequester GLUT4 away from the recycling endosomal system and to store it intracellularly until such time as insulin mobilises this fraction of GLUT4 to the cell surface.

It is well established that people with insulin resistance or Type II (non-insulin-dependent) diabetes mellitus have blunted insulin-stimulated GLUT4 translocation, presumably as a consequence of a diminished ability of insulin to recruit GLUT4 to the cell surface from intracellular stores [10, 11, 12, 13]. One potential explanation for this reduced GLUT4 translocation is that GLUT4 is incorrectly sorted within peripheral tissues, such that it no longer resides in an insulin-responsive subcellular location. Although some studies have suggested that such trafficking defects are associated with some subjects with gestational and Type II diabetes [10,11], detailed information has been hampered by an inability to biochemically resolve the distinct intracellular pools of GLUT4.

A recent study used iodixanol gradient centrifugation to analyse the subcellular distribution of GLUT4 within 3T3-L1 adipocytes and muscle [14]. With this approach, two distinct intracellular pools of GLUT4 were resolved, one which seems to be endosomal in origin and which is enriched in transferrin receptors and early endosomal rab proteins, and a second, denser fraction, which is relatively devoid of endosomal markers and which could correspond to the GLUT4 storage vesicle. The study showed that insulin stimulation results in a great loss of GLUT4 from this latter fraction, consistent with this pool being comprised of the major fraction of GLUT4 which is mobilised in response to insulin [14]. One attractive hypothesis which could explain reduced or defective GLUT4 translocation in insulin resistance or Type II diabetes is that GLUT4 is unable to gain access to the GSV compartment or that this compartment is absent.

To test this hypothesis, we have used chronically insulin treated 3T3-L1 adipocytes as an experimental model of hyperinsulinaemia [15]. Previous studies have shown that long-term treatment of 3T3-L1 adipocytes with insulin $(500 \mathrm{nmol} / \mathrm{l}$ for $24 \mathrm{~h})$ results in a partial down-regulation of total cellular GLUT4 and an inability of a subsequent short-term insulin challenge to promote GLUT4 translocation [15]. Here, we show that long-term insulin treatment of these cells results in a selective loss of GLUT4 from the GSV fraction identified by iodixanol gradient centrifugation and a concomitant reduction in insulin-stimulated GLUT4 translocation. Moreover, the targeting of the insulin-responsive aminopeptidase (IRAP) is similarly affected such that the content of this protein in the GSV fraction were greatly reduced and the protein accumulates in an endosomal compartment. Such data suggest that the mis-targeting of GLUT4 away from GSVs or the selective loss of the GSV compartment are important causal factors in the development of insulin resistance in peripheral tissues.

\section{Materials and methods}

Materials. Iodixanol was from Gibco/Life Technologies (Paisley, Scotland) and porcine insulin was generously provided by Dr G. Danielson (Novo Nordisk, Denmark). All other materials were as described previously $[16,17,18]$.

3T3-L1 adipocyte growth and differentiation. We grew 3T3-L1 fibroblasts in $10 \%$ newborn calf serum in DMEM at $37^{\circ} \mathrm{C}$ in $10 \% \mathrm{CO}_{2}$. Cells were differentiated into adipocytes as described [19] and were used 8-12 days after differentiation and between passages 4 and 12 .

Long-term insulin stimulation of 3T3-L1 adipocytes was carried out as outlined in [15]. The 3T3-L1 adipocytes were incubated with or without $500 \mathrm{nmol} / 1$ insulin for $24 \mathrm{~h}$. After this time the plates were transferred onto a $37^{\circ} \mathrm{C}$ hotplate and washed four times over the course of $80 \mathrm{~min}$ with KRM Buffer pH6 (136 mmol/1 NaCl, $4.7 \mathrm{mmol} / \mathrm{l} \mathrm{KCl}, 1.25 \mathrm{mmol} / \mathrm{l} \mathrm{CaCl}$, $1.25 \mathrm{mmol} / \mathrm{l} \mathrm{MgSO} 4,10 \mathrm{mmol} / \mathrm{l} \mathrm{MES}, 25 \mathrm{mmol} / \mathrm{l}$ Glucose, $\mathrm{pH}$ $6.0)$. The cells were quickly washed once with warm PBS, serum-free DMEM was added and cells were transferred back into an incubator at $37^{\circ} \mathrm{C}$ for $2 \mathrm{~h}$. Total membranes or subcellular fractions were then prepared as outlined below.

For plasma membrane lawn assays of GLUT4 translocation in response to short-term insulin addition, the above procedure was modified such that after incubation in serum-free DMEM for $2 \mathrm{~h}$, the plates were washed three times over $30 \mathrm{~min}$ with $\mathrm{KRH} \quad(136 \mathrm{mmol} / \mathrm{l} \mathrm{NaCl}, 4.7 \mathrm{mmol} / \mathrm{l} \mathrm{KCl}$, $1.25 \mathrm{mmol} / \mathrm{l} \mathrm{CaCl} 2,1.25 \mathrm{mmol} / \mathrm{l} \mathrm{MgSO} 4,10 \mathrm{mmol} / \mathrm{l} \mathrm{HEPES}$, $\mathrm{pH}$ 7.4) and then stimulated in this buffer with or without $1 \mu \mathrm{mol} / 1$ insulin for $30 \mathrm{~min}$ [15]. After this time subcellular fractionation, or plasma membrane lawn assays were done as outlined below.

Plasma membrane lawn assays for GLUT translocation. After experimental manipulations, coverslips of adipocytes were rapidly washed in ice-cold buffer for the preparation of plasma membrane (PM) lawns exactly as described previously [17]. Triplicate coverslips were prepared at each experimental condition and five to ten random images of plasma membrane lawns collected from each. These were quantified using MetaMorph (Universal Imaging, West Chester, Pa., USA) software as described previously [18].

Subcellular fractionation of adipocytes. Adipocytes were subjected to a differential centrifugation procedure as described previously $[6,16]$. Briefly, cells were scraped and homogenised in ice-cold HES $(20 \mathrm{mmol} / 1$ HEPES, $1 \mathrm{mmol} / \mathrm{l}$ EDTA, $250 \mathrm{mmol} / \mathrm{l}$ sucrose, $\mathrm{pH} 7.4 ; 5 \mathrm{ml}$ per $10 \mathrm{~cm}$ plate) containing complete protease inhibitor tablets (Boehringer Mannheim, Lewes, East Sussex, UK). The homogenate was subjected to a differential centrifugation procedure isolate low-density microsomal membranes or a total membrane fraction, essentially as outlined previously [17]. Briefly, for preparation of low-density microsomes (LDMs), the homogenate was centrifuged at 
$41000 \mathrm{~g}$ for $20 \mathrm{~min}$ at $4{ }^{\circ} \mathrm{C}$. The supernatant from this step was further sedimented at $180000 \mathrm{~g}$ for $1 \mathrm{~h}$ to pellet the LDM fraction. For total membranes, the homogenate was sedimented at $180000 \mathrm{~g}$ for $1 \mathrm{~h}$ to pellet total cellular membranes. For separation of LDM, high-density microsomes (HDM) and PM fractions, the homogenate was subjected to the analysis outlined previously [17].

Iodixanol gradient analysis of intracellular membranes. The LDM fraction isolated above was subjected to further analysis on OptiPrep (iodixanol: (5,5' [(2-hydroxy-1-3propanediyl) bis (acetylamino)] bis [ $N, N^{\prime}-$ bis (2,3-dihydroxypropyl2,4,6triiodo-1,3-benzenecarboxamide]; Nycodenz-Pharma AS, Oslo, Norway) gradients using a modification of the methods described previously [14]. The LDM pellet was resuspended in HES buffer and iodixanol added to $14 \%$ and gently mixed. This was then centrifuged at $295000 \mathrm{~g}$ in a near-vertical rotor (Beckman TLN 100) for $1 \mathrm{~h}$ at $4{ }^{\circ} \mathrm{C}$ and decelerated with the brakes off. Fractions of $300 \mu \mathrm{l}$ were collected from the bottom of the tube and analysed by immunoblotting as outlined. Fractions 2-4 were designated the GSV fraction and fractions 9-12 designated the TGN/endosomal fractions based upon the analysis of marker proteins (see Results and [14]).

Electrophoresis and immunoblotting. Proteins were electrophoresed on SDS-polyacrylamide gels and transblotted onto nitrocellulose as described [17]. Immunolabelled proteins were visualised using HRP-conjugated secondary antibody and quantitated by densitometry as described previously [20].

Antibodies. Antibodies to GLUT4 were those described previously [21] and antibodies against IRAP were generously provided by Drs M. Birnbaum and L. Garza (University of Pennsylvania, Pa., USA). Anti-transferrin receptor antibodies were from Zymed Laboratories (Cambridge, UK) and antivesicle-associated membrane protein 2 (VAMP2) and anti-cellubrevin serum were generously provided by Professor D. James (University of Queensland, Australia) and have been described elsewhere [6]. Anti- $\gamma$-adaptin was provided by $\mathrm{Dr}$ M. Robinson (University of Cambridge, UK) [22] and antibodies specific for the cation-dependent mannose-6-phosphate receptor by Dr A. Hille-Rhefeld (University of Göttingen, Germany).

Statistical analysis. Statistical analysis was performed using StatView software. A $p$ value of $<0.05$ was considered significant in all analyses.

\section{Results}

Iodixanol gradient centrifugation has been successfully used to resolve two distinct intracellular GLUT4 pools in 3T3-L1 adipocytes. One of these pools (fractions 9-12) is TGN/endosomal in origin and is enriched in transferrin receptors (TfR), early endosomal Rab proteins and markers for the TGN. The second, denser pool (fractions 2-4), is relatively devoid of endosomal markers and corresponds to GLUT4 storage vesicle (GSVs) [14]. Insulin stimulation results in a dramatic loss of GLUT4 from this latter pool, consistent with this being comprised of the major fraction of GLUT4 which is mobilised in response to insulin [14].
We have recapitulated this data in 3T3-L1 adipocytes (Fig.1). By this means we have resolved two distinct populations of intracellular GLUT4- and insulinresponsive aminopeptidase (IRAP)-containing vesicles. The first peak (peak 1 ) contains $40 \pm 5 \%$ of the total GLUT4 present within the LDM fraction and is designated the GSV-containing fraction. The second peak is enriched in markers for the TGN and endosomal compartments and contains $45 \pm 8 \%$ of the GLUT4 present within the LDM fraction. Consistent with the data reported previously [14], we observe a dramatic reduction in the GLUT4 content of peak 1 in response to short-term insulin stimulation $(51 \pm 3.5 \%$ reduction; Fig. 1A,B) consistent with this fraction containing the majority of the GLUT4 mobilised in response to insulin. Here, we extend the earlier studies [14] to show that insulin stimulation also resulted in a significant decrease in the IRAP content of peak 1 $(61 \pm 6.3 \%$ reduction $)$. This is consistent with GLUT4 and IRAP residing in the same intracellular compartment [23-25]. The reduction in the GLUT4 and IRAP content of peak 2 in response to insulin was significantly smaller $(23 \pm 2.6 \%$ for GLUT4, $30 \pm 4 \%$ for IRAP), in agreement with previous studies [14]. We further show that the GSV peak co-segregates with the vesicle-associated $N$-ethylmaleimide-sensitive factor attachment protein receptor (v-SNARE) VAMP2, and the TGN/endosomal peak is enriched in the v-SNARE cellubrevin (Fig.1C), consistent with published data on the co-localisation of these different v-SNAREs within different GLUT4 compartments in 3T3-L1 adipocytes [6,17,18]. Moreover, insulin-stimulation resulted in a decrease in VAMP2 content of the GSV fraction (Fig. 1C), consistent with this v-SNARE having a fundamental role in GLUT4 vesicle fusion at the plasma membrane $[17,18]$.

Treatment of 3T3-L1 adipocytes with $500 \mathrm{nmol} / \mathrm{l}$ insulin for $24 \mathrm{~h}$ resulted in the down-regulation of the total cellular GLUT4 protein content by about $40 \%$ (Fig. 2A, B) [20]. Moreover, such treatment renders these cells refractory to subsequent short-term insulin stimulation, such that a significant reduction in insulin-stimulated GLUT4 and IRAP translocation is evident, as assessed by the plasma membrane lawn assay (Fig.2C). This reduction cannot be explained solely by the reduction in GLUT4 content of the cells [15], but rather is likely to reflect both a reduced GLUT4 content and other alterations in either the translocation machinery or the insulin signalling system induced by long-term insulin treatment. Notably, long-term insulin treatment did not result in a decrease in the cellular content of IRAP (Fig.2A, B) but the extent of insulin-stimulated IRAP translocation was, like that of GLUT4, considerably reduced in long-term insulin treated cells (Fig. 2C).

We therefore sought to find whether long-term insulin treatment resulted in an alteration in the pattern of GLUT4 localisation between the two fractions 
$\begin{array}{lllllllllllll}1 & 2 & 3 & 4 & 5 & 6 & 7 & 8 & 9 & 10 & 11 & 12 & \text { Fraction }\end{array}$

GLUT-4

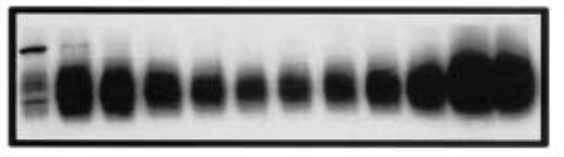

B

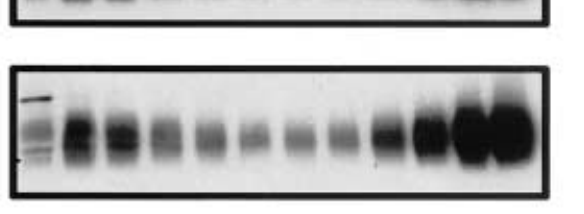

I

GLUT-4 $\begin{array}{lllllllllllll}1 & 2 & 3 & 4 & 5 & 6 & 7 & 8 & 9 & 10 & 11 & 12 & \text { Fraction }\end{array}$
IRAP

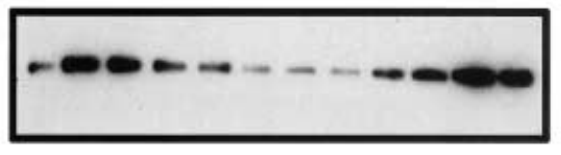

B

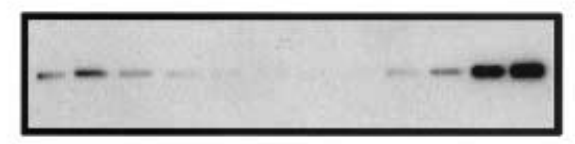

A
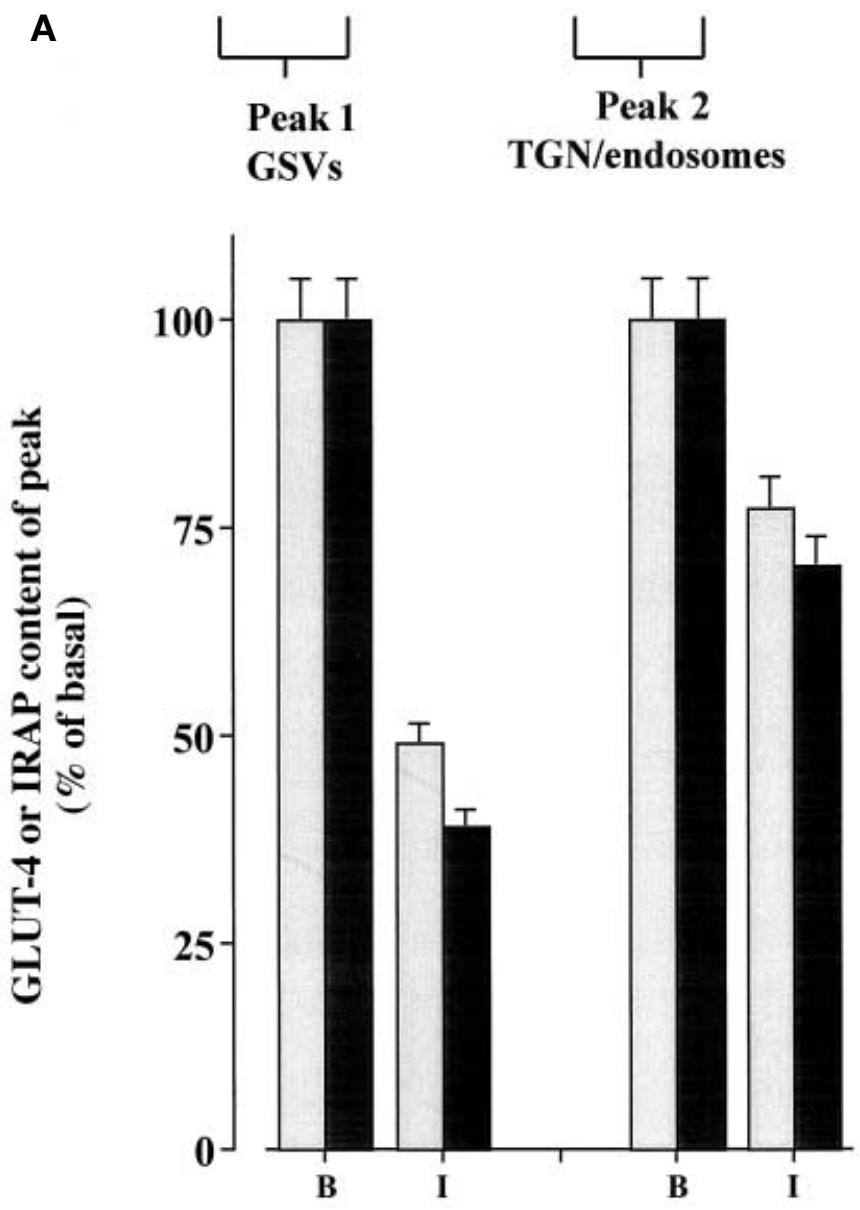

B

Peak 2

identified using iodixanol gradient centrifugation. A representative immunoblot is shown in Figure $3 \mathrm{~A}$. Analysis of the effect of long-term insulin treatment on the relative GLUT4 content of peaks 1 and 2 is presented in Figure 3B. When the amount of

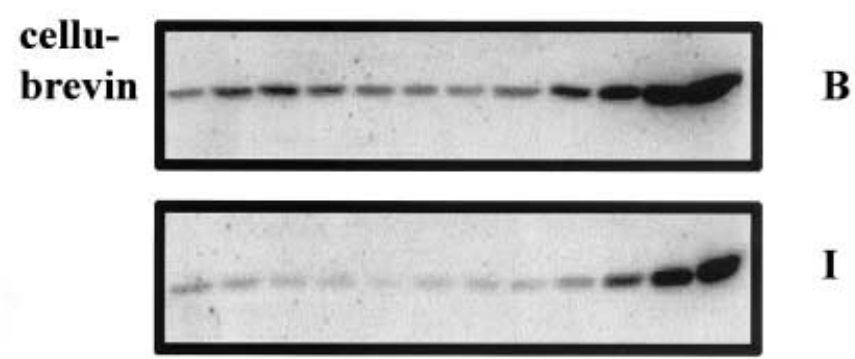

B

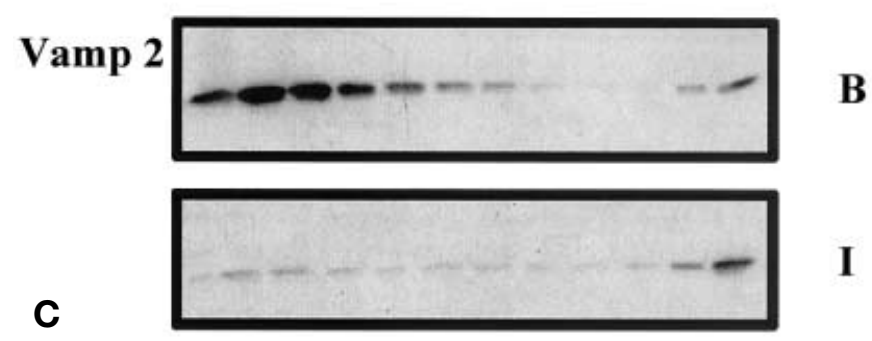

Fig. 1A-C. Iodixanol gradient sedimentation analysis intracellular membranes in 3T3-L1 adipocytes containing GLUT4, IRAP, VAMP2 and cellubrevin. 3T3-L1 adipocytes were treated with (I) or without (B) $1 \mu \mathrm{mol} / \mathrm{l}$ insulin for $30 \mathrm{~min}$ and LDM fractions subjected to iodixanol density gradient analysis. Fractions were collected from the bottom of the gradient and analysed by SDS-PAGE and immunoblotting. A typical immunoblots for GLUT4 and IRAP in basal and insulin treated cells. Peak 1 represents GLUT4 storage vesicles (GSV) and peak 2 corresponds to endosomes/TGN. Quantification of this data showed that peak 1 contained $40 \pm 5 \%$ of the total GLUT4 present within the LDM fraction of basal cells and peak 2 contained $45 \pm 8 \%$ (means $\pm \mathrm{SD}, n=4)$. B the quantification of three independent experiments of this type in which the \% change in GLUT4 ( $\square$ ) or IRAP ( $\square$ ) in each of the two peaks was quantified relative to the distribution in the absence of insulin. The greater magnitude of reduction in peak $1 \mathrm{com}$ pared to peak 2 was significant for both proteins $(p<0.05)$. C The distribution of VAMP2 and cellubrevin in the same fractions as $\mathbf{A}$. A representative immunoblot is shown

GLUT4 present in either peak are expressed as a percentage of the content in control (untreated) cells, this data clearly shows that long-term insulin treatment results in the selective loss of GLUT4 ( $68 \pm 7 \%$ reduction) from peak 1 on the iodixanol gradient. As this fraction seems to contain the majority of the insulin-sensitive GSVs, this observation suggests that long-term insulin treatment has resulted in the selective depletion of the insulin-sensitive intracellular GLUT4 pool. The depletion of GLUT4 from peak 2 was, by contrast, considerably less $(19 \pm 4.5 \%$ reduction). Several experiments of this type are quantified in Figure 3B.

In control cells, peak 1 contains $40 \pm 5 \%$ of the total GLUT4 present within the LDM fraction and peak 2 contained $45 \pm 8 \%$ of the total GLUT4 pre- 


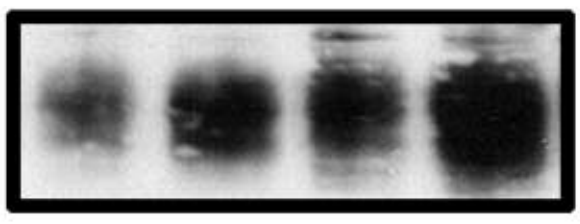

A

GLUT-4

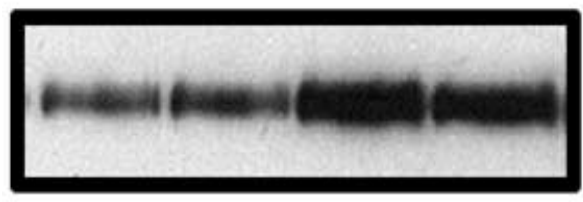

\section{IRAP}
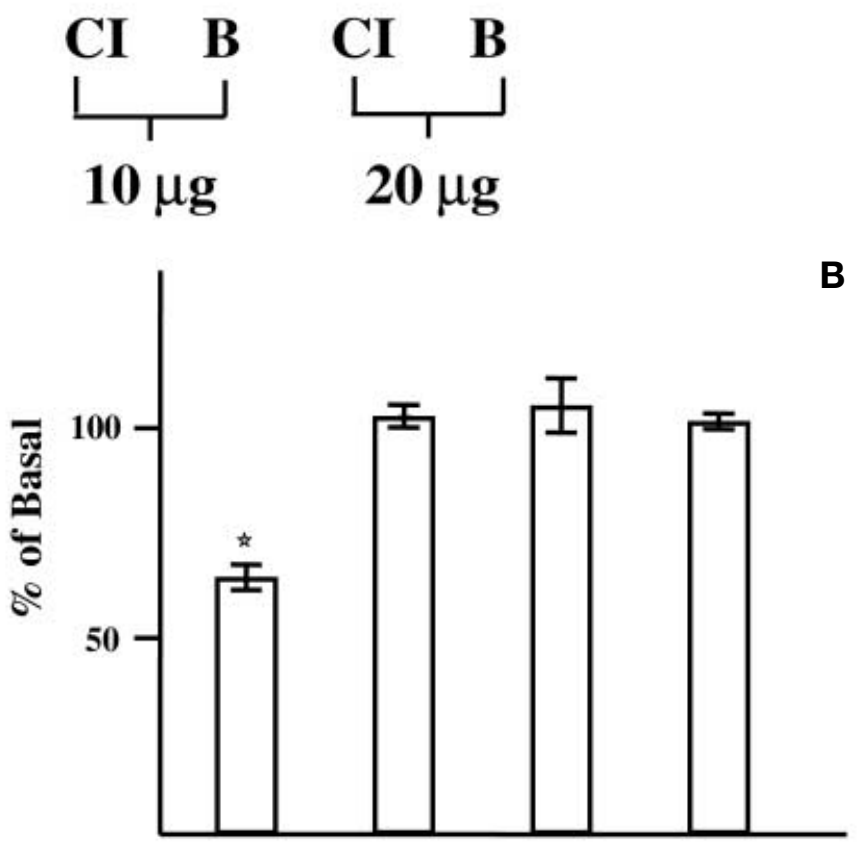

\section{GLUT-4 IRAP TfR TGN38}
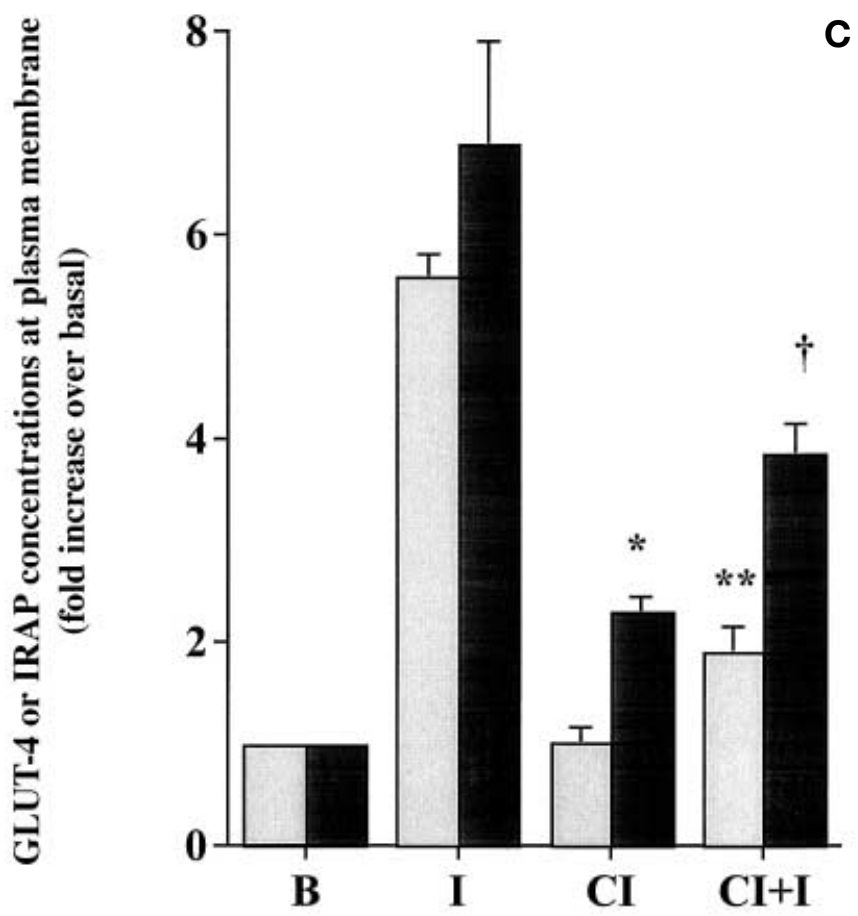

sent within the LDM fraction. After long-term insulin treatment, these values were $26 \%$ in peak 1 and $73 \%$ in peak 2 (Fig.3C). Note that the total amount of GLUT4 in the LDM fraction is about $40 \%$ lower after long-term insulin treatment, hence the fraction of total GLUT4 in peak 2 seems to increase relative to control cells. This alternative means of displaying the data further shows that in response to long-term insulin treatment, the amount of GLUT4 in peak 1 is considerably reduced and the majority of the GLUT4 present within intracellular membranes is now localised to the TGN/endosomal fraction.

We have also examined the subcellular distribution of the insulin-responsive aminopeptidase (IRAP) under the same conditions. This protein has been shown to have a high degree of co-localisation with GLUT4 in all cells studied to date [23, 26-28]. The cellular content of IRAP was not reduced by long-term insulin treatment, a result which strongly contrasts to that for GLUT4 (Fig. 2A, B), which suggests that although similarly targeted in cells, the mechanism(s) by which the cellular content and localisation of these proteins are controlled is subtly distinct. Long-term insulin treatment of 3T3-L1 adipocytes resulted in an increased amount of IRAP at the plasma membrane and a reduction in the extent of insulin-stimulated IRAP translocation (Fig.2C), as assayed using the plasma membrane lawn assay. The reduction in the magnitude of insulin-stimulated IRAP translocation is similar to that observed for GLUT4. The behaviour of IRAP is, however, distinct in that (1) the cellular content of this protein is not re-

Fig. 2A-C. IRAP and GLUT4 are differentially modulated by long-term insulin treatment and show reduced insulin responsiveness. Total membranes from control (B) and long-term insulin-treated (CI) 3T3-L1 adipocytes were prepared as outlined. A representative immunoblots for GLUT4 and IRAP in the indicated amounts of total membrane protein. B the quantification of this data and of similar analyses for transferrin receptor and TGN38. Protein content in control cells is ascribed a value of $100 \%$ and the effect of long-term insulin treatment is expressed as a \% change from this value. * indicates a significant change, $p<0.05$. C Insulin-stimulated GLUT4 ( $\square$ ) and IRAP ( $\square$ ) translocation in cells treated with or without $500 \mathrm{nmol} / \mathrm{l}$ insulin for $24 \mathrm{~h}$. After washing, cells were stimulated with $1 \mu \mathrm{mol} / \mathrm{l}$ insulin for $30 \mathrm{~min}$ and the extent of GLUT4 and IRAP translocation measured using the PM lawn assay. Shown is the fold translocation over basal from two (IRAP) and four (GLUT4) experiments (means \pm SD). B = basal cells, $\mathrm{I}=$ control cells subsequently given a short-term insulin challenge. $\mathrm{CI}=$ long-term insulin treated cells and $\mathrm{CI}+\mathrm{I}=$ longterm insulin treated cells subsequently challenged with a short-term exposure to insulin. * indicates a significant difference increase in cell surface IRAP content compared with basal cells $(\mathrm{B}), p=0.02$. $* *$ indicates a significant reduction in insulin-stimulated GLUT4 translocation compared with I, $p<0.01$ and $\dagger$ indicates a significant reduction in insulin-stimulated IRAP translocation compared with I, $p<0.05$ 
$\begin{array}{lllllllllllll}1 & 2 & 3 & 4 & 5 & 6 & 7 & 8 & 9 & 10 & 11 & 12 & \text { Fraction }\end{array}$

GLUT-4

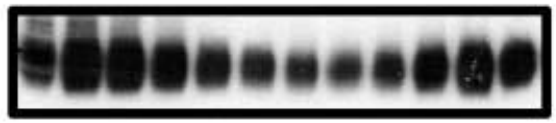

B

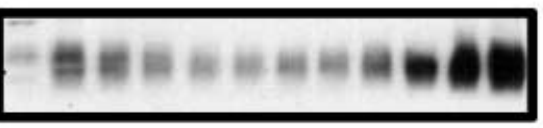

CI

IRAP

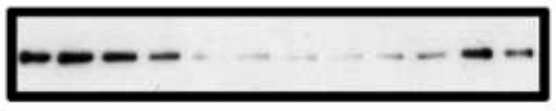

B

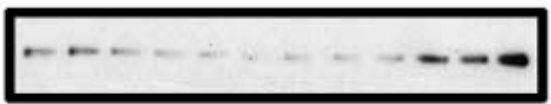

CI

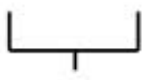

Peak 1

A

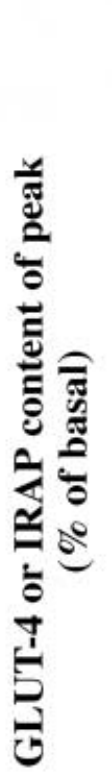

GSVs

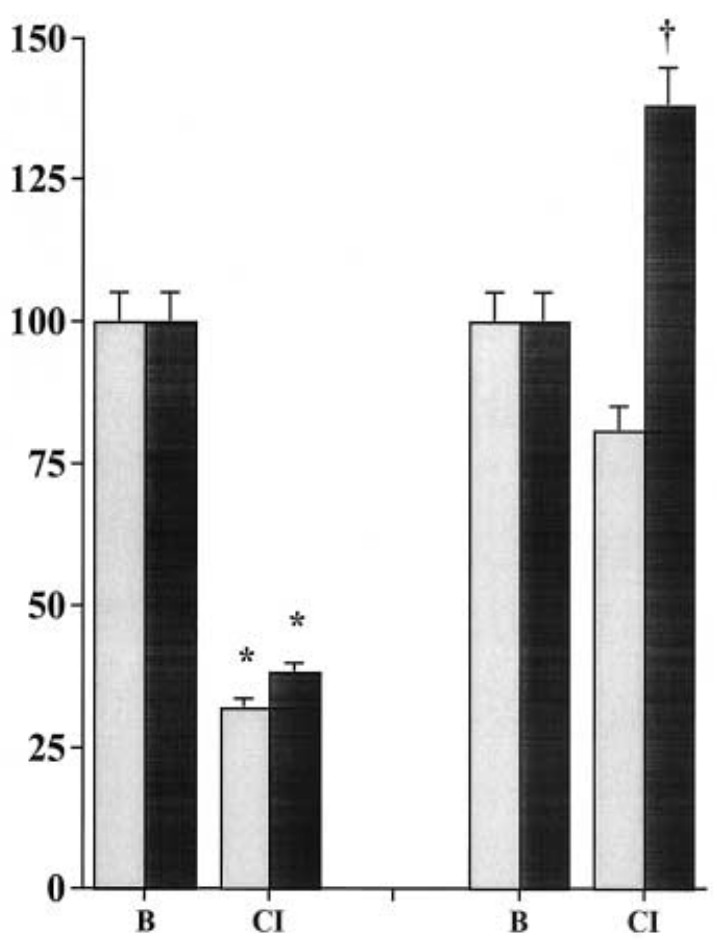

B

Peak 1

Peak 2

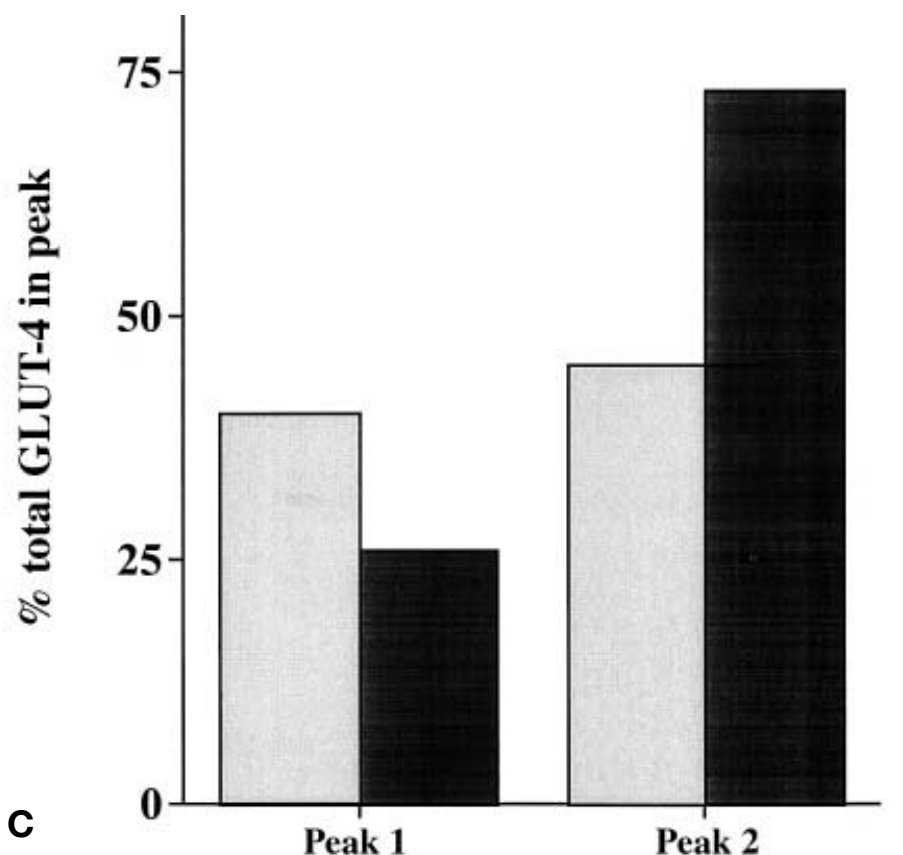

Fig.3 A-C. Iodixanol gradient sedimentation analysis of intracellular membranes from 3T3-L1 adipocytes containing GLUT4 and IRAP treated with or without long-term insulin treatment. LDM fractions from 3T3-L1 adipocytes treated with (CI) or without (B) insulin at $500 \mathrm{nmol} / \mathrm{l}$ for $24 \mathrm{~h}$ were analysed by iodixanol density gradient analysis. Fractions were collected from the bottom of the gradient and immunoblotted for GLUT4 or IRAP. A typical GLUT4 and IRAP immunoblots. B quantifies four independent experiments of this type in which the per cent change in GLUT4 ( $\square$ ) and IRAP (D) in each of the two peaks in long-term insulin-treated cells was expressed relative to that in control cells. The reduction in GLUT4 and IRAP content of peak 1 were both statistically significant $* p<0.02$. The increased amount of IRAP in peak 2 in long-term insulin cells was statistically significant compared with basal cells $\left({ }^{\dagger} p=0.04\right)$. C the changes in the GLUT4 content of peaks 1 and 2 expressed as a fraction of the total GLUT4 loaded on the iodixanol gradient. Note that under these conditions, long-term insulin-treated cells show a $40 \%$ reduction in GLUT4 content and the LDM fraction contains a correspondingly reduced GLUT4 content. Thus, longterm insulin treatment elicited a considerable redistribution of GLUT4, such that the fraction of the total present in peak 1 was reduced and that in peak 2 increased. Note that compared with control cells, the absolute GLUT4 content of peak 2 is, however, slightly reduced (see B). $\square$, basal insulin; 口, long-term insulin duced upon long-term insulin treatment (see below) and (2) the amount of IRAP at the cell surface in cells treated long-term with insulin are higher in the absence of insulin than in cells not treated with longterm insulin (Fig. 2C; see below). We have recapitulated these data using subcellular fractionation and immunoblotting. This approach also showed increased IRAP association with the plasma membrane after long-term insulin treatment (Fig. 4).

Analysis of the distribution of IRAP within intracellular membranes by iodixinal centrifugation showed that long-term insulin treatment resulted in a significant redistribution of IRAP from peak 1 to peak 2 . When expressed as a percentage change relative to control cells, there is a $62 \pm 6 \%$ reduction in peak 1 and a $38 \pm 6 \%$ increase in peak 2 (Fig. 3A, B). Hence, IRAP behaved like GLUT4 in that the IRAP was significantly reduced in the GSV compartment (peak 1), but differs from GLUT4 in that IRAP was increased in the fractions proposed to correspond to TGN/endosomes (peak 2). The increased content of IRAP in the TGN/endosomal fractions could also 

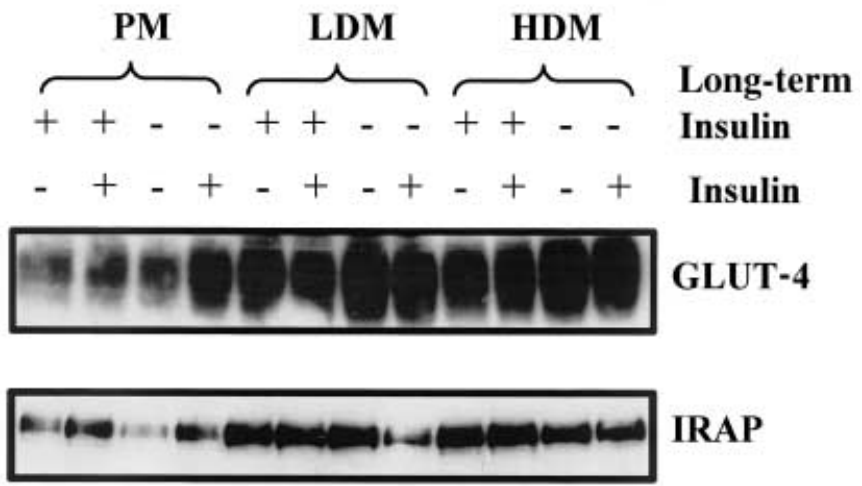

Fig. 4. Distribution of GLUT4 and IRAP in subcellular fractions. 3T3-L1 adipocytes were treated with or without insulin at $500 \mathrm{nmol} / 1$ for $24 \mathrm{~h}$, then subjected to a short-term insulin challenge ( $1 \mu \mathrm{mol} / 1$ for $30 \mathrm{~min})$ after washing. PM, LDM and HDM fractions were prepared and the distribution of GLUT4 and IRAP analysed by immunoblotting. Data from a typical experiment is presented, which was repeated with similar results. In control cells in the absence of insulin, GLUT4 was distributed 5\% in the PM fraction, 58\% in the LDM fraction and $37 \%$ in the HDM fraction

explain the higher amount of IRAP observed in the plasma membrane of cells treated long-term with insulin compared with untreated cells (see Figs. 2C, 4). The increased amount of IRAP in the TGN/endosomal fraction together with the increased plasma membrane content of IRAP account for the loss of IRAP from the GSV compartment.

To be certain that the effect of long-term insulin treatment on GLUT4 and IRAP localisation were selective, we did a series of immunoblot analyses to examine the distribution of other membrane proteins between the GSV and TGN/endosomal peaks. Figure 5 shows typical immunoblots in which the distribution of cellubrevin, VAMP2 and the AP-1 adaptor sub-unit $\gamma$-adaptin were examined. Long-term insulin treatment did not significantly reduce the cellubrevin, VAMP2 or $\gamma$-adaptin content of peak 1, arguing that the effects of long-term insulin on GLUT4 and IRAP (shown in Fig. 3) are not simply a reflection of a generalised alteration in membrane trafficking. Rather, we suggest that the effects on GLUT4 and IRAP are specific and selective. Similarly, in data not shown here, we have observed no changes in the distribution of the cation-dependent mannose-6phosphate receptor upon long-term insulin treatment. Long-term insulin treatment did, however, modestly increase the total cellular content of VAMP2, as we have described previously [20].

\section{Discussion}

Using iodixanol gradient centrifugation we have separated intracellular GLUT4 containing membranes into two distinct populations corresponding to recy- $\begin{array}{llllllllllll}1 & 2 & 3 & 4 & 5 & 6 & 7 & 8 & 9 & 10 & 11 & \text { Fraction }\end{array}$
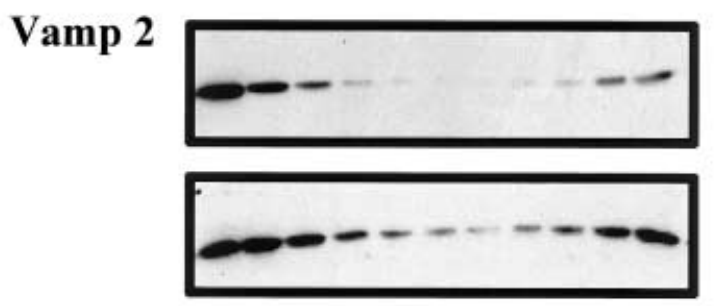

\section{cellu- brevin}

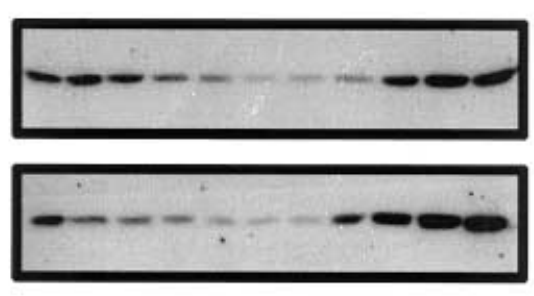

\section{AP1}

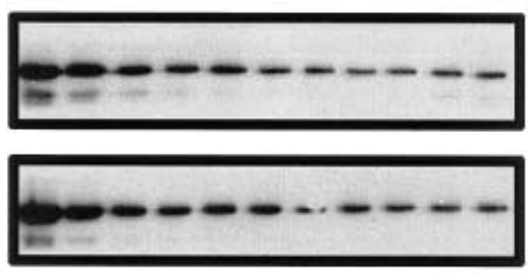

Fig. 5. Long-term insulin treatment does not significantly alter the distribution of cellubrevin, VAMP2 or $\gamma$-adaptin on iodixanol gradients. Iodixanol fractions prepared as outlined in the legend to Fig. 3 from control (B) and long-term insulin-treated (CI) cells were analysed by immunoblotting with antibodies against cellubrevin (VAMP3), VAMP2 and $\gamma$-adaptin, a component of the AP-1 complex. Shown are the data from a typical experiment. Quantification of several experiments of this type showed that there are no statistically significant changes in the distribution of these three proteins among the two peaks after long-term insulin treatment

cling endosomes and a distinct intracellular compartment which likely represents GLUT4 storage vesicles (GSVs). Long-term insulin treatment decreased cellular GLUT4 content by about $40 \%$ and diminished the ability of a short-term insulin challenge to promote GLUT4 translocation. Our data is consistent with this depletion of cellular GLUT4 arising selectively from the GSV fraction. Such data argue that long-term insulin treatment results in the mis-targeting of GLUT4 such that it no longer accesses the GSV compartment. These data imply that defective targeting of GLUT4 away from the GSV compartment may play an important role in the aetiology of insulin resistance.

We offer the following model to account for our experimental data. Long-term insulin treatment results in the mobilisation of almost all of the GSV compartment to the cell surface from where GLUT4 and IRAP gain access to the recycling endosomal system. Long-term insulin treatment bias the trafficking of these proteins such that they no longer return to 
the GSV compartment during their recycling itinerary. Cellular GLUT4 is decreased by the selective trafficking of a fraction of GLUT4 to a lysosomal pathway for degradation. By contrast, IRAP does not follow this pathway presumably as a consequence of subtle differences in the targeting signals within the cytoplasmic domains of these two proteins and the cellular content of IRAP is not depleted. In this model, the consequence of long-term insulin treatment would be reduced amounts of GLUT4 and IRAP in the GSV compartment (as shown in Fig. 3) and an increased fraction of IRAP in the recycling endosomal pathway in response to long-term insulin (Fig.3). The consequence of these perturbations would be a reduction in insulin-stimulated GLUT4 and IRAP translocation in response to short-term insulin challenge (Fig. 2C) and a modest increase in the plasma membrane content of IRAP upon long-term insulin treatment as the fraction of IRAP in the endosomal system increased (Figs. 2-4). It is important to recognise that long-term insulin treatment, in addition to the trafficking defects reported here, also has been shown to result in significant changes to both the cellular content and activities of key molecules involved in the propagation of the insulin signal $[29,30]$. Whether the changes reported here or those described in $[29,30]$ are causal or adaptive is not known. Nevertheless, our data are consistent with a defect in GLUT4 trafficking associated with long-term insulin treatment that seems to account for the reduction in insulin-stimulated GLUT4 translocation under these experimental conditions.

These data offer new insight into the trafficking of GLUT4 and IRAP in insulin-sensitive cells. The data presented are consistent with the biochemical resolution of a distinct intracellular GLUT4/IRAP-containing compartment (GSVs) which is selectively mobilised in response to insulin and further show that the down-regulation of intracellular GLUT4 known to accompany long-term insulin treatment results in the selective loss of GLUT4 from such a compartment. The data also suggest the hypothesis that mis-targeting of GLUT4 in part accompanies the development of insulin resistance. Mis-targeting of GLUT4 has been reported in some cohorts of Type II diabetic and gestational diabetic subjects $[10,11]$ the precise defect remains, however, elusive. We suggest that this mis-targeting involves an inability of GLUT4 to be correctly localised to the insulin-responsive GSV compartment. Finally, these data illustrate that the trafficking of IRAP and GLUT4 and the cellular mechanisms responsible for their homeostasis are distinct, the former presumably arising as a consequence of differences in the targeting information contained within the cytosolic domains of these two proteins.

Acknowledgements. We thank Drs Birnbaum, Danielson, Garza, Hille-Rehfeld, James and Robinson for the provision of re- agents used in this study and Drs James and Winder for critically reading the manuscript. This work was supported by a grant from the Sir Jules Thorne Charitable Trust and by equipment grants from The Wellcome Trust and Tenovus (Scotland) to G. W. Gould, and by an infrastructure grant from the Medical Research Council.

\section{References}

1. Rea S, James DE (1997) Moving GLUT4: the biogenesis and trafficking of GLUT4 storage vesicles. Diabetes 46: 1667-1677

2. Tanner LI, Lienhard GE (1987) Insulin elicits a redistribution of transferrin receptors in 3T3-L1 adipocytes through an increase in the rate constant for receptor externalisation. J Biol Chem 262: 8975-8980

3. Calderhead DM, Kitagawa K, Tanner LI, Holman GD, Lienhard GE (1990) Insulin regulation of the two glucose transporters in 3T3-L1 adipocytes. J Biol Chem 265: 13800-13808

4. Slot JW, Geuze HJ, Gigengack S, Lienhard GE, James DE (1991) Immuno-localization of the insulin regulatable glucose transporter in brown adipose tissue of the rat. J Cell Biol 113: 123-135

5. Slot JW, Garruti G, Martin S et al. (1997) Glucose transporter (GLUT-4) is targeted to secretory granules in rat atrial cardiomyocytes. J Cell Biol 137: 1243-1254

6. Martin S, Tellam J, Livingstone C, Slot JW, Gould GW, James DE (1996) The glucose transporter (GLUT4) and vesicle-associated membrane protein (VAMP2) are segregated from recycling endosomes in insulin-sensitive cells. J Cell Biol 134: 625-635

7. Livingstone C, James DE, Rice JE, Hanpeter D, Gould GW (1996) Compartment ablation analysis of the insulin responsive glucose transporter, GLUT4, in 3T3-L1 adipocytes. Biochem J 315: 487-495

8. Herman GA, Bonzelius F, Cieutat A-M, Kelly RB (1994) A distinct class of intracellular storage vesicles, identified by expression of the glucose transporter GLUT4. Proc Natl Acad Sci USA 91: 12750-12754

9. Malide D, Dwyer NK, Blanchette-Mackie EJ, Cushman SW (1997) Immunocytochemical evidence that GLUT4 resides in a specialized translocation post-endosomal VAMP2-positive compartment in rat adipose cells in the absence of insulin. J Histochem Cytochem 45: 1083-1095

10. Garvey WT, Maianu L, Zhu J-H, Hancock JA, Golichowski AM (1993) Multiple defects in the adipocyte glucose transport system cause cellular insulin resistance in gestational diabetes. Diabetes 42: 1773-1785

11. Garvey WT, Maianu L, Zhu J-H, Brechtel-Hook G, Wallace P, Baron AD (1998) Evidence for defects in the trafficking and translocation of GLUT4 glucose transporters in skeletal muscle as a cause of human insulin resistance. J Clin Invest 101: 2377-2386

12. Kahn BB (1992) Facilitative glucose transporters: regulatory mechanisms and dysregulation in diabetes. J Clin Invest 89: 1367-1374

13. Kahn BB, Rosen AS, Bak JF et al. (1992) Expression of GLUT1 and GLUT4 glucose transporters in skeletal muscle of humans with insulin-dependent diabetes mellitus: regulatory effects of metabolic factors. J Clin Endocrinol Metab 74: 1101-1109

14. Hashiramoto M, James DE (2000) Characterization of insulin-responsive GLUT4 storage vesicles isolated from 3T3-L1 adipocytes. Mol Cell Biol 20: 416-427 
15. Kozka IJ, Clark AE, Holman GD (1991) Chronic treatment with insulin selectively down-regulates cell-surface GLUT4 glucose transporters in 3T3-L1 adipocytes. J Biol Chem 266: 11726-31

16. Martin S, Reaves B, Banting G, Gould GW (1994) Analysis of the co-localization of the insulin-responsive glucose transporter (GLUT4) and the trans Golgi network marker TGN38 within 3T3-L1 adipocytes. Biochem J 300: 743-749

17. Martin L, Shewan A, Millar CA, Gould GW, James DE (1998) Vesicle associated membrane protein-2 (VAMP2) plays a specific role in the insulin-dependent trafficking of the facilitative glucose transporter GLUT4 in 3T3-L1 adipocytes. J Biol Chem 273: 1444-1452

18. Millar CA, Sherwan A, Hickson GRX, James DE, Gould GW (1999) Differential regulation of secretory compartments containing the insulin-responsive glucose transporter, GLUT4, in 3T3-L1 adipocytes. Mol Biol Cell 10: 3675-3688

19. Frost SC, Lane MD (1988) Evidence for the involvement of vicinal sulphydryl groups in insulin-activated hexose transport in 3T3-L1 adipocytes. J Biol Chem 260: 2646-2652

20. Maier V, Melvin DR, Lister CA, Chapman H, Gould GW, Murphy GJ (2000) v- and t-SNARE protein expression in models of insulin resistance: normalisation of glycemia by rosiglitazone treatment corrects over-expression of cellubrevin, VAMP2 and syntaxin 4 in skeletal muscle of Zucker Diabetic Fatty (ZDF) rats. Diabetes 49: 618-625

21. Brant AM, Jess TJ, Milligan G, Brown CM, Gould GW (1993) Immunological analysis of glucose transporters expressed in different regions of the rat brain and central nervous system. Biochem Biophys Res Commun 192: 1297-1302
22. Seaman MNJ, Ball CL, Robinson MS (1993) Targeting and mis-targeting of plasma membrane adaptors in vitro. J Cell Biol 123: 1093-1105

23. Kandror KV, Yu L, Pilch PF (1994) The major protein of GLUT4-containing vesicles, gp160, has aminopeptidase activity. J Biol Chem 269: 30777-30780

24. Kandror KV, Pilch PF (1998) Multiple endosomal recycling pathways in rat adipose cells. Biochem J 331: 829-835

25. Ross SA, Keller SR, Lienhard GE (1998) Increased intracellular sequestrtion of the insulin-regulated aminopeptidase upon differentation of 3T3-L1 cells. Biochem J 330: 1003-1008

26. Keller SR, Scott HM, Mastick CC, Aebersold R, Leinhard GE (1995) Cloning and characterisation of a novel insulinregulated membrane aminopeptidase from GLUT4 vesicles. J Biol Chem 270: 23612-23618

27. Martin S, Rice JE, Gould GW, Keller S, Slot JW, James DE (1997) The glucose transporter GLUT4 and the aminopeptidase vp165 colocalise in tubulo-vesicular elements in adipocytes and cardiomyocytes. J Cell Sci 110: 2281-2291

28. Sumitani S, Ramlal T, Somwar R, Keller SR, Klip A (1997) Insulin regulation and selective segregation with glucose transporter- 4 of the membrane aminopeptidase vp165 in rat skeletal muscle cells. Endocrinology 138: 1029-1034

29. Ricort JM, Tanti JF, Van Obberghen, Le MarchandBrustel Y (1995) Alterations in insulin signalling pathway induced by prolonged insulin treatment of 3T3-L1 adipocytes. Diabetologia 38: 1148-1156

30. Pryor PR, Liu SC, Clark AE, Holman GD, Tosh D (2000) Chronic insulin effects on insulin signalling and GLUT4 endocytosis are reversed by metformin. Biochem J 348: 83-91 